\title{
Peroxiredoxin 4 protects against ovarian ageing by ameliorating D-galactose-induced oxidative damage in mice
}

\author{
Xiuru Liang', Zhengjie Yan', Weiwei Ma', Yi Qian', Xiaofei Zou', Yugui Cui', Jiayin Liu' and Yan Meng ${ }^{1}$
}

\begin{abstract}
Peroxiredoxin 4 (Prdx4), a member of the Prdx family, is a vital ER-resident antioxidant in cells. As revealed in our previous study, Prdx4 expression was detected in ovarian granulosa cells and was closely related to ovarian function. This research aimed to explore the effect and underlying molecular mechanism of the protective role of Prdx4 against D-gal-induced ovarian ageing in mice. The D-gal-induced ovarian ageing model has been extensively used to study the mechanisms of premature ovarian failure (POF). In this study, adult Prdx $4^{-/-}$and wild-type mice were intraperitoneally injected with D-gal (150 mg/kg/day) daily for 6 weeks. Ovarian function, granulosa cell apoptosis, oxidative damage and ER stress in the ovaries were evaluated in the two groups. Ovarian weight was significantly lower, the HPO axis was more strongly disrupted, and the numbers of atretic follicles and apoptotic granulosa cells were obviously higher in $\operatorname{Prd} \times 4^{-/-}$mice. In addition, $\operatorname{Prd} \times 4^{-/-}$mice showed increased expression of oxidative damage-related factors and the ovarian senescence-related protein P16. Moreover, the levels of the proapoptotic factors CHOP and activated caspase-12 protein, which are involved in the ER stress pathway, and the level of the apoptosis-related BAX protein were elevated in the ovaries of Prd $\times 4^{-/-}$mice. Thus, D-gal-induced ovarian ageing is accelerated in Prdx4 $4^{-/-}$mice due to granulosa cell apoptosis via oxidative damage and ER stress-related pathways, suggesting that Prdx4 is a protective agent against POF.
\end{abstract}

\section{Introduction}

Infertility is a prevalent disorder worldwide and affects $15 \%$ of reproductive-aged couples ${ }^{1}$. Ovarian ageing is a major factor affecting female fertility due to depletion of ovarian follicles and loss of ovarian cyclicity ${ }^{2}$. An accumulated body of data implies that ovarian oxidative damage can result in severe ovarian dysfunctions ${ }^{3}$. Dgalactose (D-gal) treatment in mice results in excessive reactive oxygen species (ROS) production and advanced glycation end product (AGE) accumulation in the brain, myocardium, liver, kidneys, muscles, vessels and ovaries ${ }^{4-10}$. Accumulation of ROS and AGE are causes of ageing that

\footnotetext{
Correspondence: Yan Meng (ctmengyan@njmu.edu.cn)

${ }^{1}$ The State Key Laboratory of Reproductive Medicine, The Center for Clinical Reproductive Medicine, the First Affiliated Hospital of Nanjing Medical University, Nanjing, Jiangsu Province, China
}

Edited by E. Candi decrease ovarian function ${ }^{11}$. Because the accelerated ageing processes in the D-gal-induced ageing model are highly similar to those occurring during the course of ageing in humans, this model is widely used to explore the mechanisms of premature ovarian failure (POF).

Previous studies have suggested that an increased production of toxic metabolites caused by ageing-related ROS accumulation principally induces the apoptosis of ovarian granulosa cells ${ }^{12}$. Substantial amounts of data indicate that many antioxidants and antioxidant enzymes that defend cells against ROS may promote follicular development and survival ${ }^{13}$. The relationship of endoplasmic reticulum (ER) stress with oxidative stress is a vicious cycle and co-mediates apoptotic processes in cells ${ }^{14}$. ER stress is involved in granulosa cell apoptosis during follicular atresia ${ }^{15}$. Oxidative damage and ER stress are two important pathways of apoptosis that are involved 
in the occurrence and development of many ageingrelated diseases.

Peroxiredoxin $4(\operatorname{Prdx} 4)$ is an important ER-localised peroxiredoxin that eliminates $\mathrm{H}_{2} \mathrm{O}_{2}$ and promotes oxidative protein folding ${ }^{16}$. Prdx4 helps prevent steatosis progression, metabolic syndrome, inflammatory reactions and apoptotic activity by suppressing local and systemic oxidative stress. In reproduction, Prdx4 knockout leads to increased spermatogenic cell death via oxidative stress in mice ${ }^{17}$. Our published data show that Prdx4 is expressed in ovarian granulosa cells ${ }^{18}$. Moreover, we have demonstrated that $\operatorname{Prdx} 4$ expression is higher in middle-aged mice than either pubescent or aged groups of mice, and that $\operatorname{Prdx} 4$ can be detected at lower levels in ovaries from premenopausal women compared with young women ${ }^{19}$. These observations indicate that $\operatorname{Prdx} 4$ may have a protective effect on ovarian function. Additionally, the latest research with single-cell transcriptome sequencing technology analysis of ovaries from young and old cynomolgus monkeys identified Prdx4 as a new molecular marker of granulosa cell ageing, which is consistent with our results ${ }^{20}$.

However, it remains unclear if and how this factor may be involved in ovarian ageing. Considering the critical role of Prdx4 in counteracting ROS and ER stress, we postulated that Prdx4 plays a protective role against oxidative damage and ER stress in a mouse ageing model. In this study, we used D-gal to accelerate ovarian ageing to determine the effect of Prdx4 on ovarian function. Mature wild-type (WT) and Prdx4 knockout $\left(\operatorname{Prdx} 4^{-/-}\right)$mice were injected with D-gal, and ovarian function, oxidative damage-related factors, and ER stress were compared.

\section{Materials and methods}

\section{Animals and treatment}

Female C57BL/6 mice were obtained from the Nanjing Biomedical Research Institute of Nanjing University, Nanjing, China. Knockout mice were generated by the CRISPR-associated Cas9 nuclease (CRISPR/Cas9) genome editing technique ${ }^{21}$. We designed and produced two sgRNAs targeting Prdx4 exons 1-7 (E1-E7) with the CRISPR Design Tool (http://crispr.mit.edu/) developed by Massachusetts Institute of Technology (sgRNA1 forward: 5'-AGCCAGCTAAAACGGCGCGCTGG-3', reverse: 5'CCTTGATCAGTAAATATCTTTGG-3'; sgRNA2 forward: 5'-GGGGCGGAGCAAGTCTCGCGAGG-3', reverse: $5^{\prime}$-CACAATGACCTTTATTGAAGG-3'). To obtain Prdx4-positive heterozygous transgenic mice (F1 generation), F0 mice were mated with C57BL/6 mice, and neonates were examined by PCR and sequencing. Prdx $4^{-1-}$ mice were viable and could mature to give birth.

This study aimed to investigate the effect of D-galinduced acute oxidative stress on ovarian function in $\operatorname{Prdx} 4^{-/-}$and WT mice at sexual maturity. Acute oxidative stress is a widely recognised cause of ageing that leads to gradual damage to ovarian function, and D-gal-induced oxidative damage is widely used to establish mouse models of ageing to explore the mechanisms underlying ovarian ageing. In our preliminary study, no differences in physiological outcomes or ovarian function were observed between $\operatorname{Prdx} 4^{-1-}$ mice and WT mice after intraperitoneal injection of saline. Considering the limited number of Prdx $4^{-1-}$ mice, we used WT mice to determine the appropriate concentration to establish the acute oxidative stress mouse model. All 5-month-old mice were allowed to acclimatise for 1 week. Previous studies have reported the generation of POF model mice by daily intraperitoneal injections of D-gal $(200 \mathrm{mg} / \mathrm{kg} / \text { day })^{22}$. To avoid excessive stress, twenty WT C57BL/6 female mice, aged 5 months, were randomly divided into the following 4 independent groups ( 5 mice per group): saline, low-dose (D-gal, $50 \mathrm{mg} /$ $\mathrm{kg} /$ day), medium-dose (D-gal, $100 \mathrm{mg} / \mathrm{kg} /$ day) and highdose (D-gal, $150 \mathrm{mg} / \mathrm{kg} /$ day). All mice were injected daily for 42 days to determine the appropriate D-gal concentration for ovarian ageing induction.

The mice in this study were then divided into two groups of five mice per group: the WT and $\operatorname{Prdx} 4^{-1-}$ groups. Mice in these two groups were intraperitoneally injected daily with the appropriate D-gal concentration for 42 days. All experiments involving animals were approved by the Institutional Animal Care and Use Committee (IACUC) of Nanjing Medical University, and the procedures were conducted in accordance with the approved guidelines.

\section{Oestrous cycles}

Oestrous cycles were monitored by vaginal smear at 6 weeks after D-gal treatment for 12 consecutive days to evaluate the oestrous cycle stages of the mice $(n=5$ per

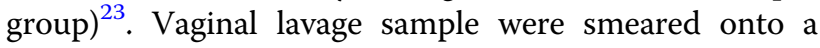
clear glass slide and stained with methylene blue. Vaginal cells were analysed by light microscopy in terms of morphological criteria. We assessed the length of each cycle as the length of time between two consecutive occurrences of oestrus and determined the time spent in each stage of the cycle.

\section{Serum preparation and hormone assays}

Blood samples were gathered by enucleation while mice were in dioestrus. Then, samples were centrifuged at $3000 \mathrm{rpm}$ for $15 \mathrm{~min}$ to collect the serum, which was stored at $-80^{\circ} \mathrm{C}$. Serum oestradiol $\left(E_{2}\right)$ and folliclestimulating hormone (FSH) levels were measured using a radioimmunoassay in the radiology department of the First Affiliated Hospital of Nanjing Medical University in accordance with the manufacturer's instructions.

\section{H\&E staining and follicle counting}

Ovaries ( $n=5$ per group) were fixed with $4 \%$ paraformaldehyde overnight and then processed for histology 
according to standard protocols. Tissues were serially sectioned $(4 \mu \mathrm{m}$ thick) throughout the entire ovary, and every fifth section was placed on a clear glass slide and stained with haematoxylin and eosin (H\&E). Follicle counting was performed as previously described with minor modifications ${ }^{24}$. In brief, to avoid counting a follicle twice, only follicles with a visible oocyte nucleus were counted. The following follicle classification system was used: primordial follicles were identified as those containing an oocyte surrounded by one layer of flattened granulosa cells; primary follicles were characterised by the presence of an enlarged oocyte surrounded by one layer of columnar granulosa cells; secondary follicles were defined as those containing more than one layer of columnar granulosa cells with no visible antrum surrounding the oocyte; antral follicles were identified as those with areas of follicular fluid or a single large antral space; and atretic follicles were identified as those exhibiting entry into a degenerative process without ovulation ${ }^{25}$. In atretic follicles, oocytes were shrunken or absent, and granulosa cells were replaced by fibrous material. The number of follicles in each category was counted by two experimenters without knowing the groups.

\section{Immunohistochemistry staining}

Sections were obtained from each mouse ovary in the two groups of mice ( $n=5$ per group) and used for immunohistochemical analysis. First, paraffin sections were dewaxed, and heat-mediated antigen retrieval was then performed by microwaving the sections in $10 \mathrm{mM}$ sodium citrate $(\mathrm{pH} 6.0)$ for $15 \mathrm{~min}$. The sections were cooled to room temperature and rinsed three times with PBS. Experimental procedures were implemented according to the manufacturer's instructions for the SP Rabbit \& Mouse HRP Kit (DAB). The samples were incubated overnight with primary antibodies (Abs) against 8-hydroxyguanosine (8-OHdG), 4-hydroxynonenal (4HNE), nitrotyrosine (NTY) and P16. Negative control samples were incubated with a biotin-conjugated goat antibody against rabbit IgG $(\mathrm{H}+\mathrm{L})$. The specimens were re-stained with haematoxylin for $30 \mathrm{~s}$. All sections were incubated simultaneously with the same antibody concentrations under the same conditions. The sections were observed and imaged by microscopy, and staining was semi-quantified with ImageJ software. Staining was quantified by measuring the immunoreactive area (IA) in $\mu \mathrm{m}^{2}$ and the integrated optical density (IOD). The staining intensity ( $\mathrm{SI}$ ) for each image was calculated as $\mathrm{SI}=$ IOD/IA.

\section{TUNEL staining}

TUNEL of apoptotic cells in ovarian tissue was detected in paraffin sections using a One Step TUNEL Apoptosis Assay Kit following the manufacturer's protocol. The sections were then counterstained with DAPI to visualise nuclei. TUNEL-positive cell counting was performed as previously described with minor modifications ${ }^{26}$. To eliminate histological differences between ovarian tissues, four random fields per slide (five slides per animal, five animals per group, $n=5$ ) were examined. In total, 100 random fields $(5 \times 5 \times 4=100)$ per group were examined. The TUNEL-positive granulosa cells and total granulosa cells in the antral follicles were counted. The rate of TUNEL-positive granulosa cells (\%) in the antral follicles was analysed using ImageJ software.

\section{Western blotting}

Western blotting was performed as described in a previous study ${ }^{27}$ In brief, protein quantification was performed using a BCA kit. Protein lysates obtained from ovarian tissues were subjected to $15 \%$ polyacrylamide gel electrophoresis and then electrotransferred to PVDF membranes. The membranes were blocked with a 5\% BSA solution for $1 \mathrm{~h}$ and then incubated with the following primary antibodies in blocking buffer overnight at $4{ }^{\circ} \mathrm{C}$ : rabbit monoclonal anti-Prdx4, rabbit polyclonal antiglucose-regulated protein 78 (GRP78), anti-activating transcription factor 4 (ATF4), anti-C/EBP homologous protein $(\mathrm{CHOP})$, anti-activating transcription factor 6 (ATF6), anti-caspase-12, rabbit monoclonal anti-BAX, rabbit polyclonal anti-superoxide dismutase 1 (SOD1), anti-superoxide dismutase 2 (SOD2), anti-catalase (CAT) and anti-GAPDH. The membranes were washed three times with TBST before incubation with HRP-conjugated secondary antibodies. The membranes were then washed three times in TBST and imaged with an Alpha Imager after development with Pierce ECL Western Blotting Substrate. For quantification of the western blot analysis results, protein levels were normalised to GAPDH levels prior to normalisation to the controls. Quantitative results were analysed using ImageJ software.

\section{Statistical analysis}

All graphs and statistical analyses were managed using GraphPad Software. Data in bar plots are shown as the mean \pm SEM and were analysed using the Student's $t$ test or one-way ANOVA. A $P$ value $<0.05$ was considered statistically significant. And the investigator was blinded to the group allocation when assessing the outcome.

\section{Results}

Establishment of the Prdx4 $4^{-1-}$ mouse model and determination of the appropriate $\mathrm{D}$-gal concentration for ovarian ageing induction

The Prdx4 gene is located on the $\mathrm{X}$ chromosome (Fig. 1a). Transcription of the Prdx4 gene generates five different transcripts, the longest of which is $100 \mathrm{bp}$ in length and contains 7 exons. To generate $\operatorname{Prdx} 4^{-1-}$ mice by 
CRISPR/Cas9-mediated genome editing, the gRNA directs Cas9 endonuclease-mediated cleavage of the $\operatorname{Prd} 4 \mathrm{4}$ gene and creates a double-strand break (DSB). Such breaks are repaired, resulting in deletion of E1-E7 (Fig. 1b). Mouse tail DNA was extracted for PCR, and ovarian tissue protein was extracted for western blotting to identify the genotypes of mice (Fig. 1c, d).

The numbers of follicles in each of the various follicle categories, the ovarian organ coefficient and the serum $\mathrm{E}_{2}$ level in the high-dose group (D-gal, $150 \mathrm{mg} / \mathrm{kg} /$ day) were significantly reduced compared with those in the control group $(P<0.05$, Fig. $1 \mathrm{e}-\mathrm{g})$. Therefore, we selected $150 \mathrm{mg} / \mathrm{kg} /$ day of D-gal as an appropriate concentration to establish the mouse model of ovarian ageing in this study.

\section{Acceleration of reproductive ageing in $\mathrm{Prdx}^{-1-}$ mice}

Analysis of the gross morphology of the two groups of mice showed no significant differences, but weight loss occurred slightly faster in the $\operatorname{Prdx} 4^{-1-}$ group than in the WT group when the POF model was induced by D-gal. Compared with the ovarian weight in the WT group, the
$\mathbf{A}$

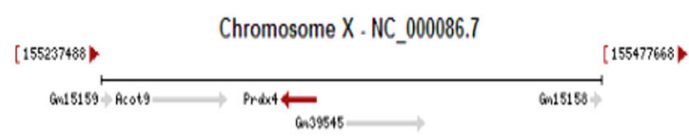

B

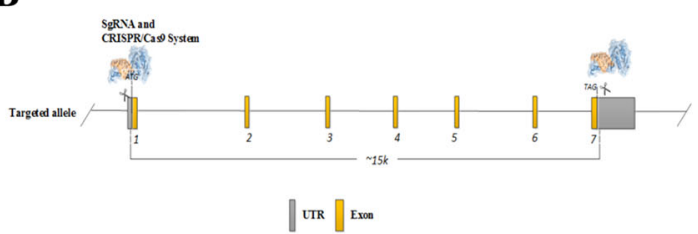

$\mathbf{E}$

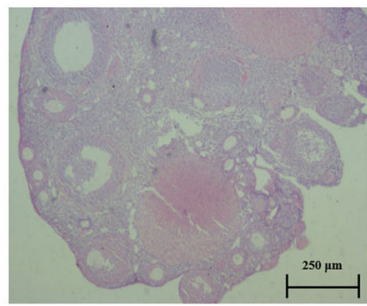

Control

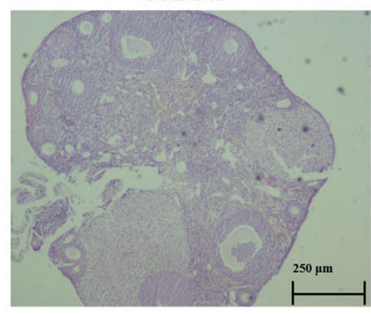

$100 \mathrm{mg} / \mathrm{kg} / \mathrm{d}$

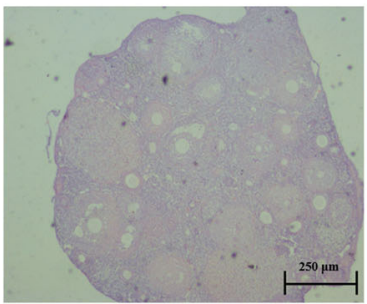

$50 \mathrm{mg} / \mathrm{kg} / \mathrm{d}$

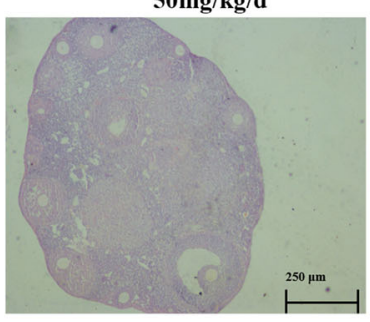

$150 \mathrm{mg} / \mathrm{kg} / \mathrm{d}$
C

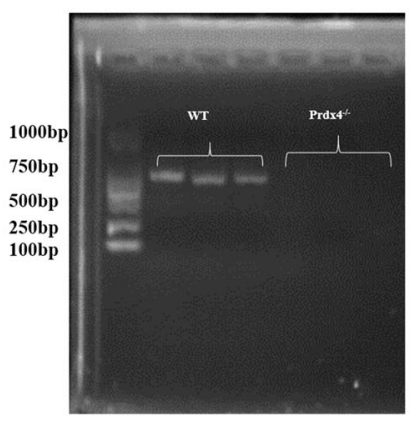

$\mathbf{F}$

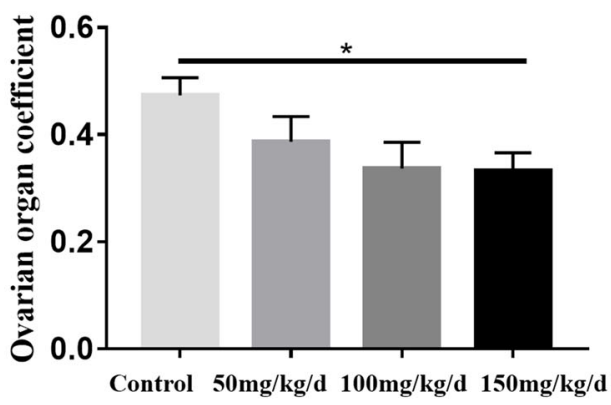

G

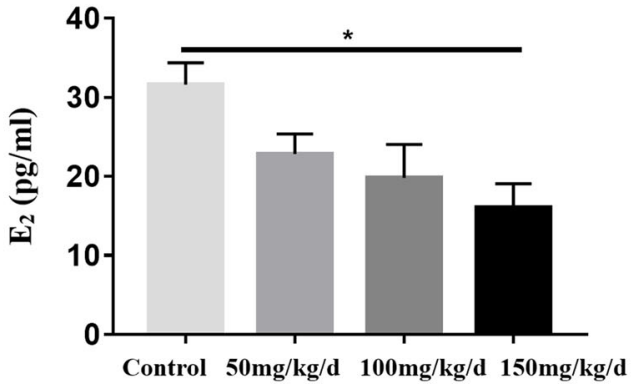

Fig. 1 Establishment of the Prdx4 $4^{-/}$mouse model and determination of the appropriate D-gal concentration for ovarian ageing induction. a The Prdx4 gene is located on the X chromosome. $\mathbf{b}$ Schematic illustration of Prdx4 exons and deletion of exons 1-7 by the CRISPR/Cas9 technique. c PCR genotyping of mice. PCR primers for the Prdx4 gene were as follows: forward, CCAGTATACAGTCTGATATGG, and reverse, TAGCTGGATATGTTAGCACT. These primers generated a 700-bp product. Genes from wild-type mice (lanes 1, 2, and 3) and Prdx4 $4^{-1-}$ mice (lanes 4, 5, and 6) were amplified with specific primers. $\mathbf{d}$ Immunoblotting analysis of Prdx4 protein expression in the control and Prdx $4^{-/-}$groups. The blot was probed with an anti-Prdx4 antibody. GAPDH was used as the internal control. e The gross morphology of the ovaries of the four groups was observed by H\&E staining. Scale bars $=250 \mu \mathrm{m}$ at $\times 4$ magnification. $\mathbf{f}$ Ovarian organ coefficient $=$ ovarian weight/body weight $(\mathrm{mg} / \mathrm{g})$. $\mathbf{g}$ Serum $E_{2}$ levels of the four groups were measured by radioimmunoassay. All data are shown as means \pm SEMs, $n=5$. Statistical significance: ${ }^{*} P<0.05$. 

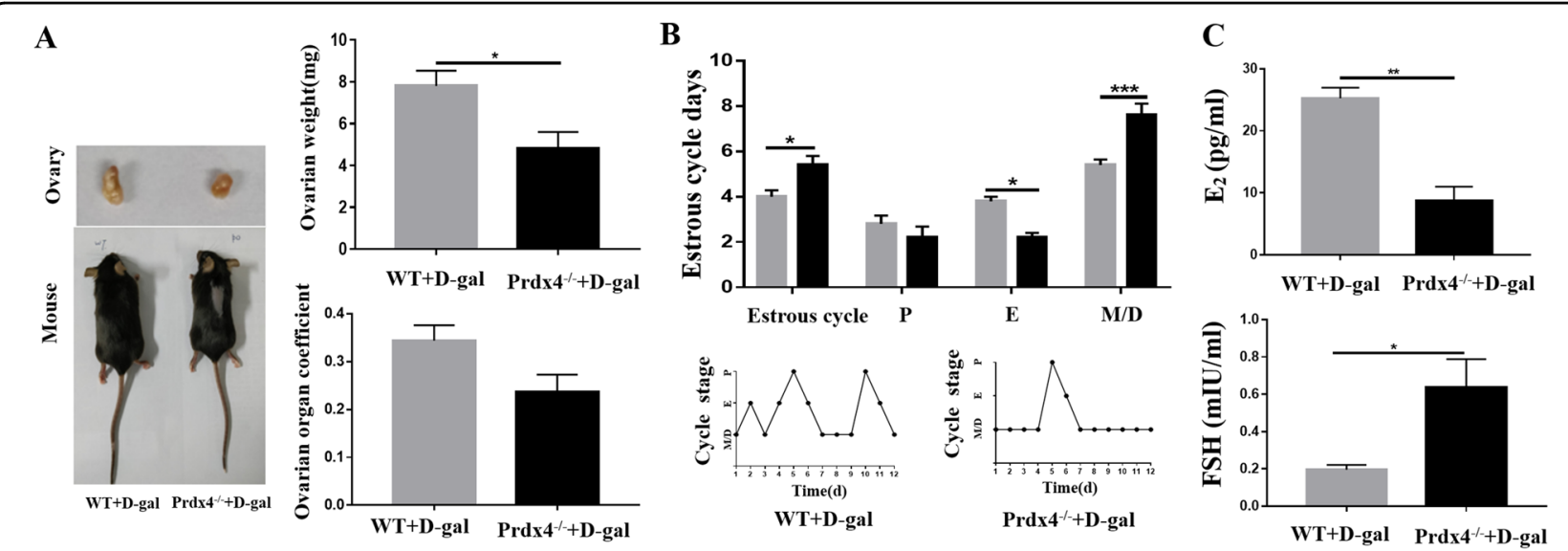

D

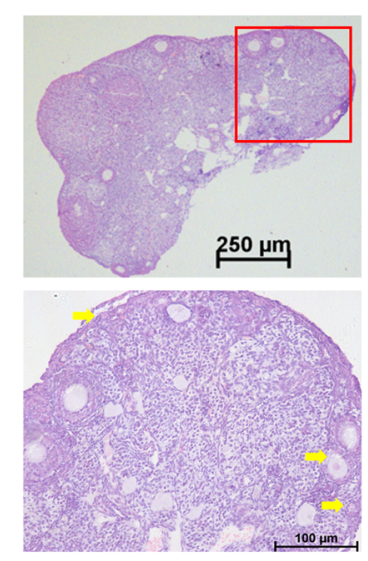

WT+D-gal

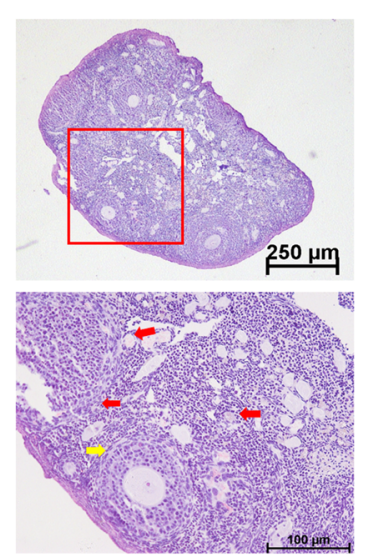

Prdx4 $4^{-/+D-g a l}$

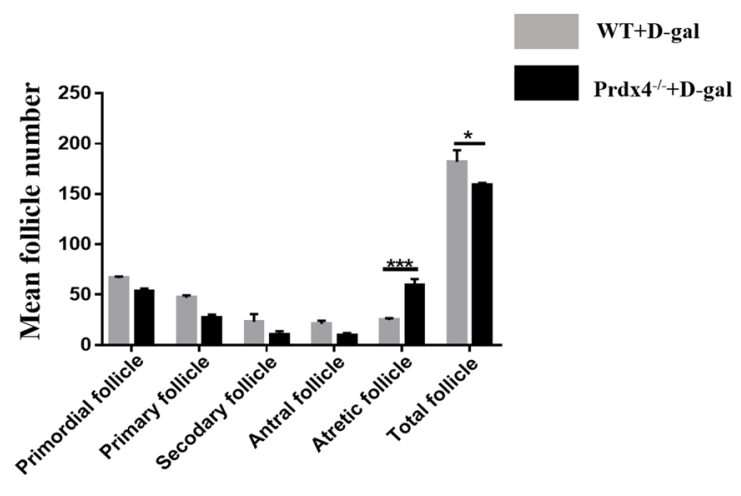

Fig. 2 Acceleration of reproductive ageing in Prdx4 ${ }^{-/-}$mice. WT and Prdx4 $4^{-1-}$ mice were injected with D-gal (150 mg/kg/d) for 42 consecutive days ( $n=5$ mice per group). a Representative images of ovaries and mice from the two groups are shown. There was a significant difference in ovarian weight between the two groups and a substantial decrease in ovarian organ coefficient in the Prdx4 ${ }^{-/-}$group. $\mathbf{b}$ The mean durations of the oestrous cycle and number of days spent in pro-oestrus $(P)$, oestrus $(E)$, metoestrus $(M)$, and dioestrus (D) were evaluated by vaginal cytology over the course of 12 consecutive days. The representative oestrous cyclicity in each treatment group is presented. c Serum $E_{2}$ and FSH levels during dioestrus were measured in the two groups. d Histology of ovarian sections from the two groups. The yellow arrows show the primordial, primary, or secondary follicles, and the red arrows indicate the developmentally arrested follicles with degenerating oocytes. Scale bars $=250 \mu \mathrm{m}$ at $\times 4$ magnification; scale bar $=100 \mu \mathrm{m}$ at $\times 20$ magnification. The number of follicles at different developmental stages is summarised. All data are shown as means \pm SEMs, $n=5$. Statistical significance: ${ }^{*} P<0.05,{ }^{* *} P<0.01,{ }^{* * *} P<0.001$.

ovarian weight in the $\operatorname{Prd} \times 4^{-1-}$ group was significantly decreased $(P<0.05)$, but the ovarian organ coefficient was not significantly decreased (Fig. 2a). The hypothalamicpituitary-ovary (HPO) axis was more strongly disrupted in the $\operatorname{Prdx} 4^{-1-}$ group than in the WT group. Oestrous cyclicity was examined via a daily vaginal smear. The results showed that the control group exhibited regular oestrous cycles with a duration of 4-5 days; however, the $\operatorname{Prd} 4^{-1-}$ group showed irregular oestrous cycles with an obviously decreased length of the oestrous period and an increased length of the dioestrus period (Fig. 2b). We then collected mouse sera to analyse the levels of $E_{2}$ and FSH, and the $\mathrm{Prdx}^{-/-}$group showed a significant decrease in the serum $\mathrm{E}_{2}$ level $(P<0.01$, Fig. $2 \mathrm{c})$ and an increase in the serum FSH level $(P<0.05$, Fig. $2 c)$. Follicle counting was performed after $\mathrm{H} \& \mathrm{E}$ staining. In $\operatorname{Prdx} 4^{-1-}$ mice, the numbers of primordial, primary, secondary and antral follicles were relatively reduced, but the number of atretic follicles was obviously increased $(P<0.001$, Fig. 2 d).

\section{Increased ovarian cell apoptosis in Prdx4 ${ }^{-1-}$ mice}

We observed visible green-stained nuclei of TUNELpositive (apoptotic) granulosa cells in antral follicles but not in the primordial and primary follicles (Fig. 3a). The number of apoptotic granulosa cell areas was assessed in the two groups with ImageJ software. More apoptotic cells were detected in the Prdx4 $4^{-1-}$ group than in the control group $(P<0.05$, Fig. $3 \mathrm{~b})$. 


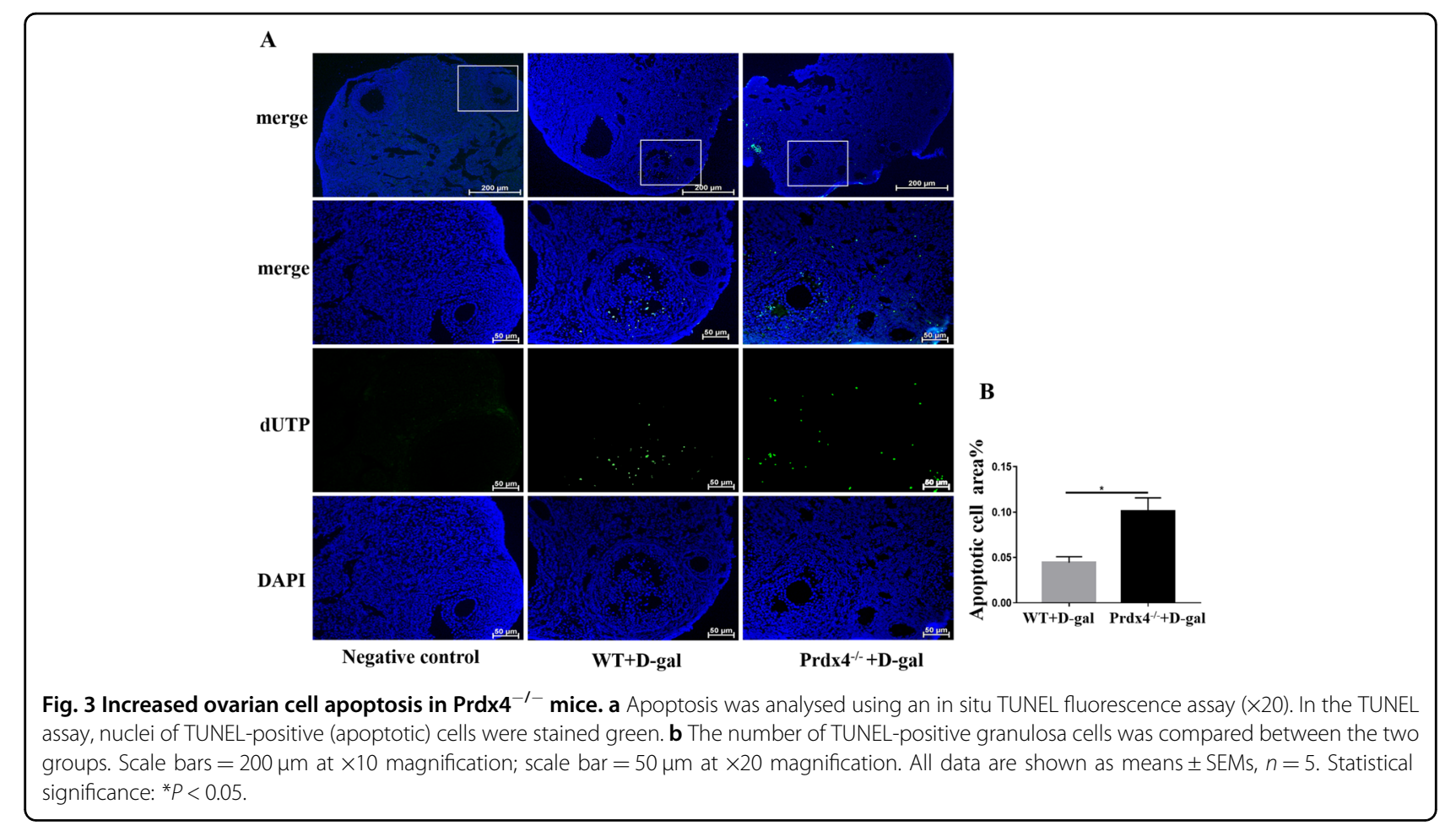

Increased oxidative damage and ovarian senescenceassociated protein expression in $\mathrm{Prdx} 4^{-/-}$mice

Oxidative DNA, protein, and lipid damage and the level of the senescence-associated protein P16 in interstitial cells and follicles were examined using immunohistochemistry. The 8-OHdG, NTY and 4-HNE proteins were localised mainly in ovarian interstitial cells. Compared with the control group, the Prdx $4^{-/-}$group showed significant increases in 8-OHdG, NTY and 4-HNE protein immunostaining $(P<0.05$, Fig. $4 \mathrm{a}, \mathrm{b})$. The ovarian senescence-associated $\mathrm{P} 16$ protein was localised mainly in granulosa cells and oocytes, and its expression levels were relatively decreased in interstitial cells. Significantly increased P16 expression was detected in the Prdx $4^{-1-}$ mouse group $(P<0.01$, Fig. $4 \mathrm{a}, \mathrm{b})$.

The expression of compensatory antioxidant enzymes and ER stress pathway-associated factors in the ovary

In Prdx $4^{-1-}$ mice, the levels of oxidative damage-related factors were visibly increased, as assessed by immunohistochemistry. In addition, the expression of other compensatory antioxidant enzymes (CAT, SOD1 and SOD2) was examined by western blotting (Fig. 5a). CAT and SOD1 protein expression levels were relatively higher in Prdx4 $4^{-/-}$mice than in WT mice, and the SOD2 protein expression level was significantly higher in $\operatorname{Prdx} 4^{-/-}$ mice $(P<0.05$, Fig. $5 c)$. Prdx 4 was localised to the ER as determined by colocalization with calreticulin ${ }^{16}$. The expression levels of ER stress signalling pathway- associated markers were assessed by western blotting (Fig. 5b, d). The expression level of an ER stress marker (GRP78) was relatively increased in the $\operatorname{Prdx} 4^{-1-}$ group. We examined the regulation of 2 major components of the unfolded protein response (UPR) pathways, namely, ATF6 and ATF4, and found that the ATF4 expression level was significantly increased $(P<0.05)$. The expression levels of CHOP/GADD153 and caspase-12 (regulators and markers of ER stress-induced apoptosis) were significantly increased $(P<0.05)$. Upregulation of the proapoptotic gene BAX $(P<0.05)$ induced apoptosis in the $\operatorname{Prd} \times 4^{-1-}$ mouse group.

\section{Discussion}

Many studies have shown that D-gal directly induces oxidative stress in vivo and that galactose toxicity inhibits $\mathrm{E}_{2}$ production from granulosa cells ${ }^{28}$. In this study, we found that the level of serum $E_{2}$ and the numbers of primordial, primary and secondary follicles were significantly decreased in mice treated with D-gal (Fig. 1e, g). Thus, the use of D-gal successfully induced the mouse POF model in the present study. The D-gal-treated mouse ageing model in this research not only partially simulated ageing-related physiological senescence in the body, but also facilitates an understanding of the pathogenesis of ovarian ageing caused by a large amount of oxidative stress. Many factors, including iatrogenic factors, harmful environments and some pathological events, can cause a sharp decline in ovarian function due to oxidative 


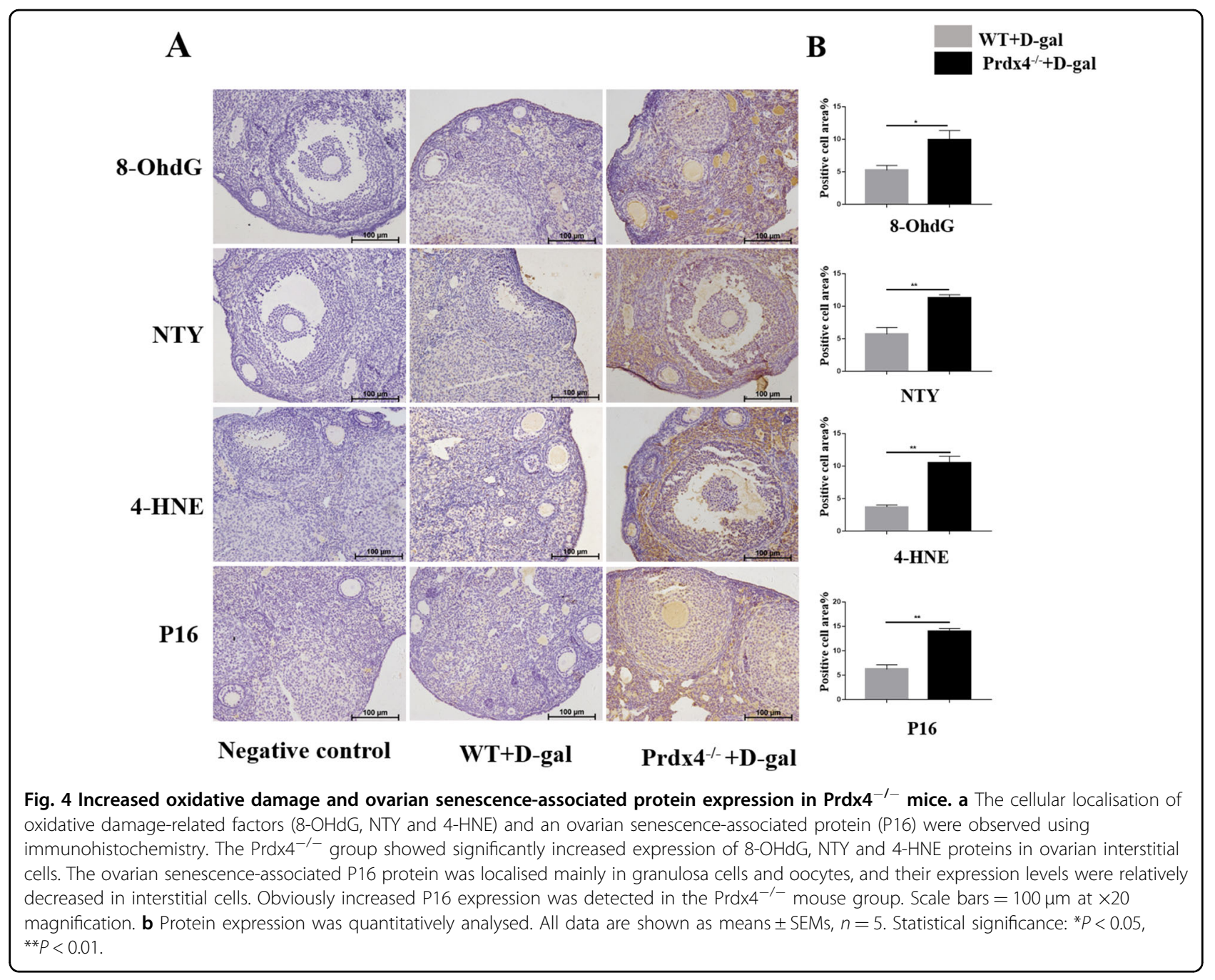

damage. Therapeutic processes such as radiotherapy and chemotherapy in cancer patients and ischaemia and reperfusion during ovarian surgery impair the ovaries through oxidative stress-induced apoptosis of follicular cells $^{29}$. Exposure to many toxic chemicals, such as those in cigarettes and pesticides, polycyclic aromatic hydrocarbons and cypermethrin, in residential and occupational environments reduces ovarian function by generating additional ROS in vivo ${ }^{30}$. Obesity and abnormal lipid metabolism are associated with infertility and high oxidative levels in the ovarian microenvironment, which may play a role in the pathogenesis of disease-related infertility $^{31}$. These forms of acute and severe oxidative damage may cause a rapid decline in ovarian function and lead to a POF. Therefore, accelerating ovarian ageing with D-gal is of practical significance.

$\operatorname{Prdx} 4$ is an antioxidant, anti-steatosis, antiinflammatory and anti-apoptotic substance ${ }^{32}$. As revealed by our previous work, Prdx4 expression closely follows follicular development ${ }^{19}$. This study was designed to investigate the protective effect of $\operatorname{Prdx} 4$ on ovarian ageing induced by D-gal. Ovarian ageing is a major factor in female infertility and characterised by decreased follicular quantity and quality ${ }^{33}$. Our data showed that with Dgal treatment, Prdx4 deficiency accelerated ovarian ageing, including increased follicular atresia and HPO axis disorders. Many studies strongly support the idea that antral follicular atresia is caused mainly by granulosa cell apoptosis $^{34}$. Granulosa cells are essential for follicle development and homeostasis because they provide nutrients and mechanical support for oocytes via physical interactions. As revealed in our previous work, Prdx4 expression was mainly located in granulosa cells. We also detected increased apoptosis of ovarian granulosa cells in $\operatorname{Prd} 4^{-1-}$ mice (Fig. 3), leading to an increase in follicle attrition and a decrease in steroid hormone production ${ }^{35}$.

To our knowledge, mammalian cells contain six peroxiredoxins, but only Prdx4 is localised to the ER. Prdx4 exerts two advantageous effects in the ER: promotion of protein disulphide bond synthesis and suppression of 


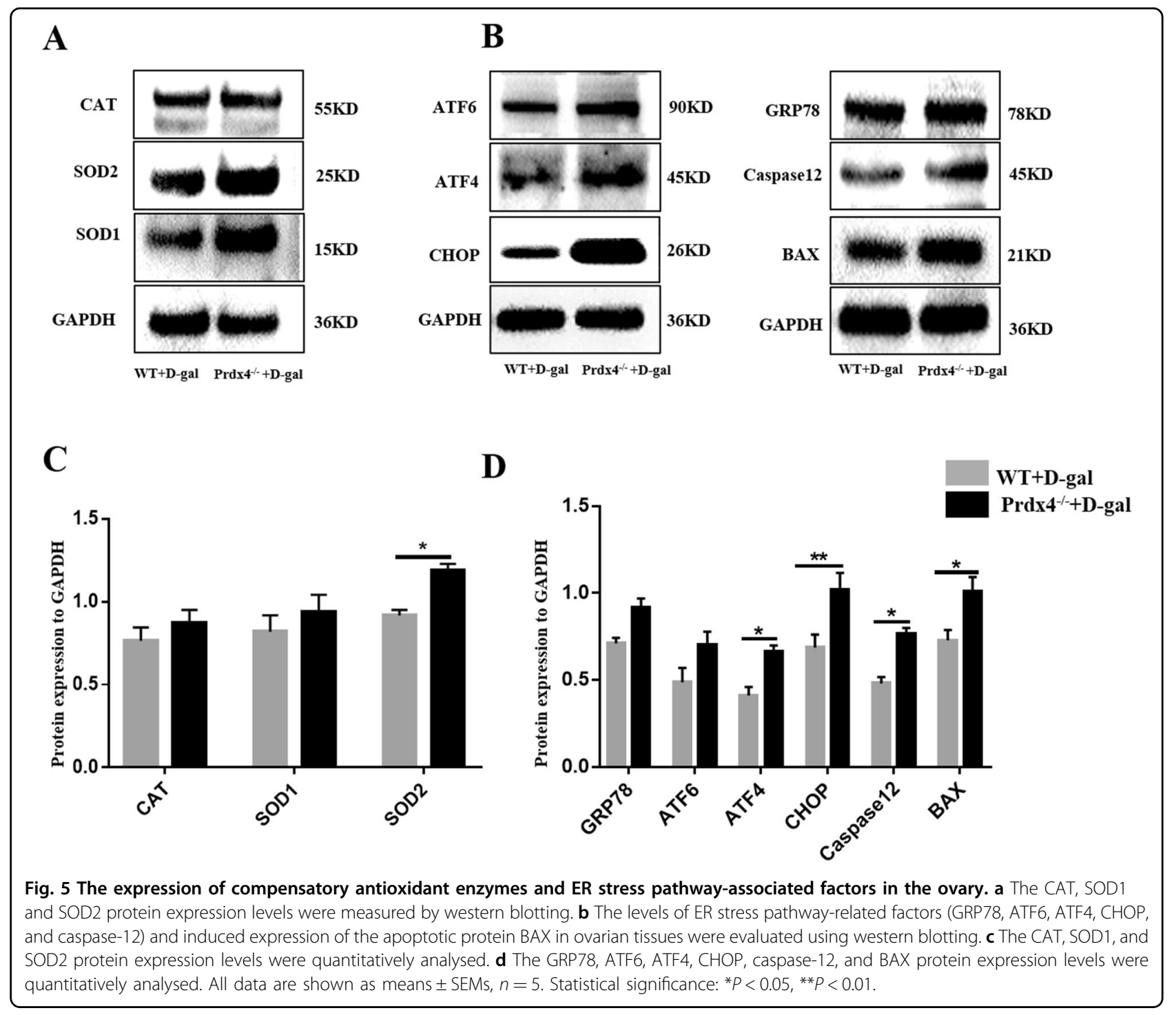

oxidative stress by eliminating $\mathrm{H}_{2} \mathrm{O}_{2}{ }^{36}$. Prdx 4 shows very strong reactivity with $\mathrm{H}_{2} \mathrm{O}_{2}$ in the ER lumen ${ }^{37}$, and excessive production of $\mathrm{H}_{2} \mathrm{O}_{2}$ is thought to lead to oxidative stress. Excess ROS lead to a wide range of oxidative damage in cell structures, including lipid peroxidation and DNA damage as well as membrane and protein oxidation, ultimately driving cell death ${ }^{38}$. Additionally, Prdx4 deficiency leads to increases in misfolded proteins and $\mathrm{H}_{2} \mathrm{O}_{2}$ in the ER lumen, inducing ER stress $^{39}$. Furthermore, studies have demonstrated that when using a dextran sulphate sodium (DSS)-induced colitis model, lack of Prdx4 aggravates the colonic mucosal damage caused by ER stress ${ }^{40}$. Prdx 4 functions as an ER thiol oxidase and antioxidant and protects the homeostasis of the cell environment. In our study, the alterations of oxidative damage- and ER-stress-related biomarkers confirmed that when all mature mice were treated with D-gal, Prdx4 deficiency increased unfavourable ROS-mediated sulfhydryl oxidation in proteins and triggered ER stress. The evidence strongly indicates that ROS resulting from exposure to specific chemical or physical agents are involved in the initiation of granulosa cell apoptosis ${ }^{41}$. Many studies have demonstrated that severe and persistent ER stress plays a crucial role in regulating GC cell apoptosis ${ }^{42}$. Thus, we concluded that Prdx4 may protect against ovarian ageing by reducing ovarian granulosa cell apoptosis via inhibiting oxidative stress and ER stress-related pathways. Although the specific mechanism has not been fully elaborated, these results provide further evidence supporting the significant role of $\operatorname{Prdx} 4$ in protecting ovarian function from ROS damage and ER stress.

Interestingly, we found that P16 expression in follicles was significantly increased in $\operatorname{Prdx} 4^{-1-}$ mice (Fig. 4). 
P16 protein negatively regulates cell proliferation and division and promotes apoptosis and senescence. And, it affects the cell cycle and G1-S conversion by competitively suppressing the interaction between CDK4/6 and cyclin D within G1 phase ${ }^{43}$. The P16 expression level was positively related to the follicular number and negatively related to $\mathrm{E}_{2}$ and $\mathrm{P}$ levels ${ }^{28}$. P16 expression was low in young animal tissues and subsequently increased with age $^{44}$. Baker et al. ${ }^{45}$ found that aging cells were deactivated and ageing-associated phenotypes reduced by silencing P16 protein expression. However, how P16 participates in ovarian aging through oxidative stress is unclear. We observed an important effect of P16 on oxidative stress in ovarian follicles and speculated that Prdx4 deficiency also accelerated ovarian ageing partially through the upregulation of P16.

In summary, our current findings suggest that Prdx4 deficiency accelerates ovarian ageing, including increased follicle atresia and HPO axis disorders, in mice exposed to D-gal-induced oxidative damage. $\operatorname{Prdx} 4$ may protect against ovarian ageing by suppressing ovarian granulosa cell apoptosis via multiple mechanisms, including inhibiting oxidative stress and ER stress-related pathways. These results provide an understanding of the pathogenesis of ovarian ageing caused by a large amount of oxidative stress. Based on the downregulated Prdx4 expression observed in premenopausal ovaries in our previous clinical study, we will further clarify the role of Prdx4 in sustaining female reproduction to reveal new diagnostic biomarkers and potential therapeutic targets for use in patients with POF.

\section{Acknowledgements}

This work was supported by the National Key Research and Development Program of China (2017YFC1001300), the National Science Foundation of China (81200439), Natural Science Foundation Jiangsu Province of China (BK20161592), Merck China Research Fund for Fertility Experts (MerckSeronoCREATE-2016023) and Jiangsu Province High-level Health Personnel Project (LGY2019066).

\section{Conflict of interest}

The authors declare that they have no conflict of interest.

\section{Publisher's note}

Springer Nature remains neutral with regard to jurisdictional claims in published maps and institutional affiliations.

Supplementary Information accompanies this paper at (https://doi.org/ 10.1038/s41419-020-03253-8).

Received: 8 February 2020 Revised: 5 November 2020 Accepted: 13 November 2020

Published online: 11 December 2020

\footnotetext{
References

1. Zhou, Z. et al. Epidemiology of infertility in China: a population-based study. BJOG 125, 432-441 (2018).
}

2. Gosden, R. G., Laing, S. C., Felicio, L. S., Nelson, J. F. \& Finch, C. E. Imminent oocyte exhaustion and reduced follicular recruitment mark the transition to acyclicity in aging C57BL/6J mice. Biol. Reprod. 28, 255-260 (1983).

3. Pandey, A. K. et al. Impact of stress on female reproductive health disorders: possible beneficial effects of shatavari (Asparagus racemosus). Biomed. Pharmacother. 103, 46-49 (2018)

4. Kimura, T. et al. Accumulation of advanced glycation end products of the Maillard reaction with age in human hippocampal neurons. Neurosci. Lett. 208, 53-56 (1996).

5. Simm, A. et al. Age associated changes of AGE-receptor expression: RAGE upregulation is associated with human heart dysfunction. Exp. Gerontol. 39, 407-413 (2004).

6. Hyogo, H. \& Yamagishi, S. Advanced glycation end products (AGEs) and their involvement in liver disease. Curr. Pharm. Des. 14, 969-972 (2008).

7. Schinzel, R., Munch, G., Heidland, A. \& Sebekova, K. Advanced glycation end products in end-stage renal disease and their removal. Nephron 87, 295-303 (2001).

8. Haus, J. M., Carrithers, J. A., Trappe, S. W. \& Trappe, T. A. Collagen, cross-linking, and advanced glycation end products in aging human skeletal muscle. J. Appl. Physiol. 103, 2068-2076 (2007).

9. Nerlich, A. G. \& Schleicher, E. D. N(epsilon)-(carboxymethyl)lysine in atherosclerotic vascular lesions as a marker for local oxidative stress. Atherosclerosis 144, 41-47 (1999).

10. Bandyopadhyay, S. et al. Galactose toxicity in the rat as a model for premature ovarian failure: an experimental approach readdressed. Hum. Reprod. 18, 2031-2038 (2003).

11. Semba, R. D., Nicklett, E. J. \& Ferrucci, L. Does accumulation of advanced glycation end products contribute to the aging phenotype? J. Gerontol. A Biol. Sci. Med. Sci. 65, 963-975 (2010).

12. Tatone, C. \& Amicarelli, F. The aging ovary-the poor granulosa cells. Fertil. Steril. 99, 12-17 (2013).

13. Devine, P. J., Perreault, S. D. \& Luderer, U. Roles of reactive oxygen species and antioxidants in ovarian toxicity. Biol. Reprod. 86, 27 (2012).

14. Malhotra, J. D. \& Kaufman, R. J. Endoplasmic reticulum stress and oxidative stress: a vicious cycle or a double-edged sword? Antioxid. Redox Signal. 9, 2277-2293 (2007).

15. Lin, P. et al. Endoplasmic reticulum stress is involved in granulosa cell apoptosis during follicular atresia in goat ovaries. Mol. Reprod. Dev. 79, 423-432 (2012).

16. Zito, E. PRDX4, an endoplasmic reticulum-localized peroxiredoxin at the crossroads between enzymatic oxidative protein folding and nonenzymatic protein oxidation. Antioxid. Redox Signal. 18, 1666-1674 (2013).

17. luchi, Y. et al. Peroxiredoxin 4 knockout results in elevated spermatogenic cell death via oxidative stress. Biochem. J. 419, 149-158 (2009).

18. Meng, Y. et al. The protein profile of mouse mature cumulus-oocyte complex. Biochim. Biophys. Acta 1774, 1477-1490 (2007).

19. Qian, Y. et al. Implication of differential Peroxiredoxin 4 expression with age in ovaries of mouse and human for ovarian aging. Curr. Mol. Med. 16, 243-251 (2016).

20. Wang, S. et al. Single-cell transcriptomic atlas of primate ovarian aging. Cell 180, 585-600.e519 (2020).

21. Yang, $\mathrm{H}$. et al. One-step generation of mice carrying reporter and conditional alleles by CRISPR/Cas-mediated genome engineering. Cell 154, 1370-1379 (2013).

22. He, L., Ling, L., Wei, T., Wang, Y. \& Xiong, Z. Ginsenoside Rg1 improves fertility and reduces ovarian pathological damages in premature ovarian failure model of mice. Exp. Biol. Med. 242, 683-691 (2017).

23. Goldman, J. M., Murr, A. S. \& Cooper, R. L. The rodent estrous cycle: characterization of vaginal cytology and its utility in toxicological studies. Birth Defects Res. B Dev. Reprod. Toxicol. 80, 84-97 (2007).

24. Bernal, A. B., Vickers, M. H., Hampton, M. B., Poynton, R. A. \& Sloboda, D. M. Maternal undernutrition significantly impacts ovarian follicle number and increases ovarian oxidative stress in adult rat offspring. PLoS ONE 5, e15558 (2010).

25. Borgeest, C., Symonds, D., Mayer, L. P., Hoyer, P. B. \& Flaws, J. A. Methoxychlor may cause ovarian follicular atresia and proliferation of the ovarian epithelium in the mouse. Toxicol. Sci. 68, 473-478 (2002).

26. Yan, Z. et al. Curcumin exerts a protective effect against premature ovarian failure in mice. J. Mol. Endocrinol. 60, 261-271 (2018). 
27. Banerjee, S. et al. Ovotoxic effects of galactose involve attenuation of folliclestimulating hormone bioactivity and up-regulation of granulosa cell p53 expression. PLOS ONE 7, e30709 (2012).

28. Attema, J. L., Pronk, C. J., Norddahl, G. L., Nygren, J. M. \& Bryder, D. Hematopoietic stem cell ageing is uncoupled from p16 INK4A-mediated senescence. Oncogene 28, 2238-2243 (2009).

29. Kececi, M., Akpolat, M., Gulle, K., Gencer, E. \& Sahbaz, A. Evaluation of preventive effect of shilajit on radiation-induced apoptosis on ovaries. Arch. Gynecol. Obstet. 293, 1255-1262 (2016).

30. Wang, $\mathrm{H}$. et al. Cypermethrin exposure reduces the ovarian reserve by causing mitochondrial dysfunction in granulosa cells. Toxicol. Appl. Pharmacol. 379 114693 (2019).

31. Silvestris, E., Lovero, D. \& Palmirotta, R. Nutrition and female fertility: an interdependent correlation. Front. Endocrinol. 10, 346 (2019).

32. Yamada, S. \& Guo, X. Peroxiredoxin 4 (PRDX4): Its critical in vivo roles in animal models of metabolic syndrome ranging from atherosclerosis to nonalcoholic fatty liver disease. Pathol. Int. 68, 91-101 (2018).

33. Shi, L. et al. Long-term moderate oxidative stress decreased ovarian reproductive function by reducing follicle quality and progesterone production. PLOS ONE 11, e0162194 (2016).

34. Asselin, E., Xiao, C. W., Wang, Y. F. \& Tsang, B. K. Mammalian follicular development and atresia: role of apoptosis. Biol. Signals Recept. 9, 87-95 (2000).

35. Luderer, U. Ovarian toxicity from reactive oxygen species. Vitam. Horm. 94 99-127 (2014).
36. Tavender, T. J. \& Bulleid, N. J. Peroxiredoxin IV protects cells from oxidative stress by removing $\mathrm{H} 2 \mathrm{O} 2$ produced during disulphide formation. J. Cell Sci. 123, 2672-2679 (2010).

37. Szarka, A. \& Lorincz, T. The role of ascorbate in protein folding. Protoplasma 251, 489-497 (2014).

38. Avila, J., Gonzalez-Fernandez, R., Rotoli, D., Hernandez, J. \& Palumbo, A. Oxidative stress in granulosa-lutein cells from in vitro fertilization patients. Reprod. Sci. 23, 1656-1661 (2016).

39. Zito, E., Hansen, H. G., Yeo, G. S., Fujii, J. \& Ron, D. Endoplasmic reticulum thiol oxidase deficiency leads to ascorbic acid depletion and noncanonical scurvy in mice. Mol. Cell 48, 39-51 (2012).

40. Takagi, T. et al. Elevated ER stress exacerbates dextran sulfate sodium-induced colitis in PRDX4-knockout mice. Free Radic. Biol. Med. 134, 153-164 (2019).

41. Bhardwaj, J. K. \& Saraf, P. Malathion-induced granulosa cell apoptosis in caprine antral follicles: an ultrastructural and flow cytometric analysis. Microsc Microanal. 20, 1861-1868 (2014).

42. Huang, N., Yu, Y. \& Qiao, J. Dual role for the unfolded protein response in the ovary: adaption and apoptosis. Protein Cell 8, 14-24 (2017).

43. Turner, N. C. et al. Palbociclib in hormone-receptor-positive advanced breast cancer. New Engl. J. Med. 373, 209-219 (2015).

44. Hsu, Y. M. \& Yin, M. C. EPA or DHA enhanced oxidative stress and aging protein expression in brain of d-galactose treated mice. Biomedicine 6, 17 (2016).

45. Baker, D. J. et al. Clearance of p16lnk4a-positive senescent cells delays ageingassociated disorders. Nature 479, 232-236 (2011). 